\title{
SOME IDENTITIES INVOLVING THE PARTITION FUNCTION
}

\author{
ODDMUND KOLBERG
}

1. Introduction. In a paper concerning certain arithmetical properties of $p(n)$, the number of unrestricted partitions of $n$, Ramanujan [13] stated without proof the identities

$$
\begin{gathered}
\sum_{n=0}^{\infty} p(5 n+4) x^{n}=5 \frac{\left\{\left(1-x^{5}\right)\left(1-x^{10}\right)\left(1-x^{15}\right) \ldots\right\}^{5}}{\left\{(1-x)\left(1-x^{2}\right)\left(1-x^{3}\right) \ldots\right\}^{6}}, \\
\sum_{n=0}^{\infty} p(7 n+5) x^{n}=7 \frac{\left\{\left(1-x^{7}\right)\left(1-x^{14}\right) \ldots\right\}^{3}}{\left\{(1-x)\left(1-x^{2}\right) \ldots\right\}^{4}}+49 x \frac{\left\{\left(1-x^{7}\right)\left(1-x^{14}\right) \ldots\right\}^{7}}{\left\{(1-x)\left(1-x^{2}\right) \ldots\right\}^{8}} .
\end{gathered}
$$

The first proofs were given by Darling [4] and Mordell [9] respectively. See also Watson [14], Rademacher and Zuckerman [12], Rademacher [11], Kruyswijk [7], Bailey [2] [3], Newman [10] and Fine [5].

Other identities involving the partition function have been found by Watson [14], Zuckerman [15], Rademacher [11], Lehner [8] and Fine [5]. Atkin and Swinnerton-Dyer [1] have deduced several remarkable congruences for the moduli 5, 7 and 11 .

In the following we shall prove some identities which seem to be new. We also give new proofs of the Ramanujan identities and four of the congruences of Atkin and Swinnerton-Dyer.

2. Definitions and lemmas. We use the notation

$$
\varphi(x)=\prod_{n=1}^{\infty}\left(1-x^{n}\right) .
$$

Then we have the well-known identities (with $p(0)=1$ )

$$
\begin{aligned}
& \varphi(x)^{-1}=\sum_{n=0}^{\infty} p(n) x^{n} \\
& \varphi(x)=\sum_{n=-\infty}^{\infty}(-1)^{n} x^{\frac{1}{2} n(3 n+1)}
\end{aligned}
$$

Received April 10, 1957. 


$$
\varphi(x)^{3}=\sum_{n=0}^{\infty}(-1)^{n}(2 n+1) x^{\frac{1}{2} n(n+1)}
$$

In the following $q$ always denotes a prime. Now let $q$ be given. We then define, for $s=0,1, \ldots, q-1$ :

$$
\begin{aligned}
& g_{s}=\sum_{\frac{1}{2} n(3 n+1) \equiv s(\bmod q)}(-1)^{n} x^{\frac{1}{2} n(3 n+1)}, \\
& h_{s}=\sum_{\substack{\frac{1}{2} n(n+1) \equiv s(\bmod q) \\
n \geqq 0}}(-1)^{n}(2 n+1) x^{\frac{1}{2} n(n+1)},
\end{aligned}
$$

that is, we split up the power series into $q$ parts, such that each part consists of those terms whose exponents belong to the same residue class $(\bmod q)$.

Lemma 1. If $24 s+1$ is a quadratic non-residue $(\bmod q)$, then $g_{s}=0$. If $24 s+1 \equiv 0(\bmod q)$, then

$$
g_{s}=(-1)^{\left[\frac{1}{8}(q+1)\right]} x^{\frac{1}{2 \frac{4}{4}}\left(q^{2}-1\right)} \varphi\left(x^{q^{2}}\right) .
$$

Proof. We have

$$
24 \cdot \frac{1}{2} n(3 n+1)+1=(6 n+1)^{2} .
$$

Hence, if $24 s+1$ is a quadratic non-residue $(\bmod q)$, we have for all $n$

and therefore $g_{s}=0$.

$$
\frac{1}{2} n(3 n+1) \neq s(\bmod q),
$$

Now let $24 s+1 \equiv 0(\bmod q)$. Then $g_{s}$ consists of those terms in (2.2) where

$$
24 \cdot \frac{1}{2} n(3 n+1)+1 \equiv 0(\bmod q),
$$

that is

Obviously, $q>3$. Let

$$
6 n+1 \equiv 0 \quad(\bmod q) .
$$

$$
n_{0}=\frac{1}{6}(-1 \pm q) \text {, }
$$

where the upper sign is used when $q \equiv 1(\bmod 6)$, the lower sign when $q \equiv-1(\bmod 6)$. Now the general solution of $(2.6)$ can be written

Thus we have

$$
n=q k+n_{0}, \quad k=0, \pm 1, \pm 2, \ldots
$$

$$
\begin{aligned}
g_{s} & =\sum_{k=-\infty}^{\infty}(-1)^{q k+n_{0}} x^{\frac{1}{2}\left(q k+n_{0}\right)\left(3 q k+3 n_{0}+1\right)} \\
& =(-1)^{n_{0}} x^{\frac{1}{2} n_{0}\left(3 n_{0}+1\right)} \sum_{k=-\infty}^{\infty}(-1)^{k} x^{\frac{1}{2} k(3 k \pm 1) q^{2}} \\
& =(-1)^{\left[\frac{1}{(q+1)]} x^{\frac{1}{24}\left(q^{2}-1\right)} \varphi\left(x^{q^{2}}\right) .\right.}
\end{aligned}
$$


Lemma 2. If $8 s+1$ is a quadratic non-residue $(\bmod q)$, then $h_{s}=0$. If $8 s+1 \equiv 0(\bmod q)$, then

$$
h_{s}=(-1)^{\frac{1}{2}(q-1)} q x^{\frac{1}{8}\left(q^{2}-1\right)} \varphi\left(x^{q^{2}}\right)^{3} .
$$

Proof. The first part of the lemma follows immediately from the identity

$$
8 \cdot \frac{1}{2} n(n+1)+1=(2 n+1)^{2} .
$$

Let $8 s+1 \equiv 0(\bmod q)$. Then we have to solve the congruence

$$
8 \cdot \frac{1}{2} n(n+1)+1 \equiv 0 \quad(\bmod q),
$$

where now $n \geqq 0$. (Obviously, $q>2$ ). The solution is

Hence

$$
n=q k+\frac{1}{2}(q-1), \quad k=0,1,2, \ldots
$$

We define

$$
\begin{aligned}
h_{s} & =\sum_{k=0}^{\infty}(-1)^{q k+\frac{1}{2}(q-1)}(2 q k+q) x^{\frac{1}{2}\left\{q k+\frac{1}{2}(q-1)\right\}\left\{q k+\frac{1}{2}(q+1)\right\}} \\
& =(-1)^{\frac{1}{2}(q-1)} q x^{\frac{1}{8}\left(q^{2}-1\right)} \sum_{k=0}^{\infty}(-1)^{k}(2 k+1) x^{\frac{1}{2} k(k+1) q^{2}} \\
& =(-1)^{\frac{1}{2}(q-1)} q x^{\frac{1}{(}\left(q^{2}-1\right)} \varphi\left(x^{q^{2}}\right)^{3} .
\end{aligned}
$$

$$
\begin{aligned}
D & =\left|\begin{array}{lll}
g_{0} & g_{1} \ldots & g_{q-1} \\
g_{q-1} & g_{0} \ldots & g_{q-2} \\
\ldots & \ldots & \ldots \\
g_{1} & g_{2} \ldots & g_{0}
\end{array}\right|, \\
D_{s} & =\left|\begin{array}{llll}
g_{-s} & g_{-s+1} & \ldots & g_{-s+q-2} \\
g_{-s-1} & g_{-s} & \ldots & g_{-s+q-3} \\
\ldots \ldots & \ldots \ldots & \ldots & \ldots \\
g_{-s-q+2} & g_{-s-q+3} & \ldots & g_{-s}
\end{array}\right|, \quad s=0,1, \ldots, q-1,
\end{aligned}
$$

where we have to put $g_{r}=g_{s}$ when $r \equiv s(\bmod q)$.

Lemma 3. We have

$$
D=\frac{\varphi\left(x^{q}\right)^{q+1}}{\varphi\left(x^{q^{2}}\right)} .
$$

Proof. Let $\omega=e^{2 \pi i / q}$. Then, by a well-known theorem we have

$$
D=\prod_{k=0}^{q-1}\left(g_{0}+\omega^{k} g_{1}+\ldots+\omega^{(q-1) k} g_{q-1}\right)
$$


But from the definition of $g_{s}=g_{s}(x)$ it follows that

and hence

$$
\omega^{s k} g_{s}(x)=g_{s}\left(\omega^{k} x\right),
$$

$$
\begin{aligned}
g_{0}+\omega^{k} g_{1}+\ldots & +\omega^{(q-1) k} g_{q-1} \\
& =g_{0}\left(\omega^{k} x\right)+g_{1}\left(\omega^{k} x\right)+\ldots+g_{q-1}\left(\omega^{k} x\right)=\varphi\left(\omega^{k} x\right) .
\end{aligned}
$$

Now, returning to $(2.7)$ we get

$$
\begin{aligned}
D & =\prod_{k=0}^{q-1} \varphi\left(\omega^{k} x\right) \\
& =\prod_{n=1}^{\infty} \prod_{k=0}^{q-1}\left(1-\omega^{k n} x^{n}\right) \\
& =\prod_{n \neq 0}\left(1-x^{q n}\right) \prod_{n \equiv 0(\bmod q)}\left(1-x^{n}\right)^{q},
\end{aligned}
$$

and lemma 3 follows.

Lemma 4. We have, for $s=0,1, \ldots, q-1$,

$$
\sum_{n=0}^{\infty} p(q n+s) x^{q n+s}=(-1)^{(q-1) s} \frac{\varphi\left(\boldsymbol{x}^{q^{2}}\right)}{\varphi\left(x^{q}\right)^{q+1}} D_{s} .
$$

Proof. Let

$$
P_{s}=\sum_{n=0}^{\infty} p(q n+s) x^{q n+s} .
$$

Now we have

$$
\begin{aligned}
P_{0}+P_{1}+\ldots+P_{q-1} & =\varphi(x)^{-1}, \\
g_{0}+g_{1}+\ldots+g_{q-1} & =\varphi(x) .
\end{aligned}
$$

By multiplication we obtain

$$
\begin{aligned}
& g_{0} P_{0}+g_{q-1} P_{1}+\ldots+g_{1} P_{q-1}=1, \\
& g_{1} P_{0}+g_{0} P_{1}+\ldots+g_{2} P_{q-1}=0, \\
& \ldots \ldots \ldots+\ldots \ldots+\cdots+\cdots+g_{0} P_{q-1}=0 . \\
& g_{q-1} P_{0}+g_{q-2} P_{1}+\ldots .
\end{aligned}
$$

From this we easily find

$$
P_{s}=(-1)^{(q-s) s} D^{-1} D_{s}
$$

and lemma 4 follows. 
3. The case $q=3$. We shall prove the identities

$$
\begin{aligned}
\sum_{n=0}^{\infty} p(3 n) x^{n}=\frac{\varphi\left(x^{3}\right) \varphi\left(x^{9}\right)^{2}}{\varphi(x)^{4}} \prod_{m=1}^{\infty}\left\{\left(1-x^{9 m-5}\right)\left(1-x^{9 m-4}\right)\right\}^{2}- \\
-x \frac{\varphi\left(x^{9}\right)^{2}}{\varphi(x)^{3}} \prod_{m=1}^{\infty}\left\{\left(1-x^{9 m-5}\right)\left(1-x^{9 m-4}\right)\right\}^{-1}
\end{aligned}
$$

$$
\begin{aligned}
\sum_{n=0}^{\infty} p(3 n+1) x^{n}= & \frac{\varphi\left(x^{9}\right)^{2}}{\varphi(x)^{3}} \prod_{m=1}^{\infty}\left\{\left(1-x^{9 m-8}\right)\left(1-x^{9 m-1}\right)\right\}^{-1}+ \\
& +x \frac{\varphi\left(x^{3}\right) \varphi\left(x^{9}\right)^{2}}{\varphi(x)^{4}} \prod_{m=1}^{\infty}\left\{\left(1-x^{9 m-8}\right)\left(1-x^{9 m-1}\right)\right\}^{2}
\end{aligned}
$$

$$
\begin{aligned}
\sum_{n=0}^{\infty} p(3 n+2) x^{n}=\frac{\varphi\left(x^{9}\right)^{2}}{\varphi(x)^{3}} \prod_{m=1}^{\infty}\left\{\left(1-x^{9 m-7}\right)\left(1-x^{9 m-2}\right)\right\}^{-1}+ \\
+\frac{\varphi\left(x^{3}\right) \varphi\left(x^{9}\right)^{2}}{\varphi(x)^{4}} \prod_{m=1}^{\infty}\left\{\left(1-x^{9 m-7}\right)\left(1-x^{9 m-2}\right)\right\}^{2}
\end{aligned}
$$

$$
\begin{aligned}
\sum_{n=0}^{\infty} p(3 n) x^{n} \sum_{n=0}^{\infty} p(3 n+1) x^{n} \sum_{n=0}^{\infty} & p(3 n+2) x^{n} \\
& =2 \frac{\varphi\left(x^{3}\right) \varphi\left(x^{9}\right)^{3}}{\varphi(x)^{7}}+9 x \frac{\varphi\left(x^{3}\right) \varphi\left(x^{9}\right)^{\mathbf{6}}}{\varphi(x)^{\mathbf{1 0}}} .
\end{aligned}
$$

Proofs. Lemma 4 yields

$$
\sum_{n=0}^{\infty} p(3 n+s) x^{3 n+s}=\frac{\varphi\left(x^{9}\right)}{\varphi\left(x^{3}\right)^{4}} D_{s},
$$

where

$$
\left\{\begin{array}{l}
D_{0}=g_{0}^{2}-g_{1} g_{2} \\
D_{1}=g_{2}^{2}-g_{0} g_{1} \\
D_{2}=g_{1}{ }^{2}-g_{2} g_{0}
\end{array}\right.
$$

In this case the conditions of lemma 1 are not fulfilled, but we can find expressions for $g_{0}, g_{1}$ and $g_{2}$ by using the following well-known identity of Jacobi:

$$
\sum_{k=-\infty}^{\infty} y^{k} z^{k^{2}}=\varphi\left(z^{2}\right) \prod_{m=1}^{\infty}\left\{\left(1+y z^{2 m-1}\right)\left(1+y^{-1} z^{2 m-1}\right)\right\} .
$$


Now, the congruence $\frac{1}{2} n(3 n+1) \equiv 0(\bmod 3)$ has the solution $n=3 k$, $k=0, \pm 1, \pm 2, \ldots$ Hence

$$
g_{0}=\sum_{k=-\infty}^{\infty}(-1)^{3 k} x^{\frac{1}{2} \cdot 3 k(9 k+1)}=\sum_{k=-\infty}^{\infty}(-1)^{k} x^{\frac{3}{2} k+\frac{27}{2} k^{2}} .
$$

Putting $y=-x^{3 / 2}, z=x^{27 / 2}$, we get from (3.7)

$$
g_{0}=\varphi\left(x^{27}\right) \prod_{m=1}^{\infty}\left\{\left(1-x^{27 m-15}\right)\left(1-x^{27 m-12}\right)\right\} \text {. }
$$

Similarly we find

$$
\begin{aligned}
& g_{1}=-x \varphi\left(x^{27}\right) \prod_{m=1}^{\infty}\left\{\left(1-x^{27 m-21}\right)\left(1-x^{27 m-6}\right)\right\} \\
& g_{2}=-x^{2} \varphi\left(x^{27}\right) \prod_{m=1}^{\infty}\left\{\left(1-x^{27 m-24}\right)\left(1-x^{27 m-3}\right)\right\} .
\end{aligned}
$$

Further, by multiplication

$$
g_{0} g_{1} g_{2}=x^{3} \frac{\varphi\left(x^{3}\right) \varphi\left(x^{27}\right)^{3}}{\varphi\left(x^{9}\right)} .
$$

Now we have

$$
\begin{aligned}
D_{0}=g_{0}^{2}-\frac{g_{0} g_{1} g_{2}}{g_{0}}= & \varphi\left(x^{27}\right)^{2} \prod_{m=1}^{\infty}\left\{\left(1-x^{27 m-15}\right)\left(1-x^{27 m-12}\right)\right\}^{2}- \\
& -x^{3} \frac{\varphi\left(x^{3}\right) \varphi\left(x^{27}\right)^{2}}{\varphi\left(x^{9}\right)} \prod_{m=1}^{\infty}\left\{\left(1-x^{27 m-15}\right)\left(1-x^{27 m-12}\right)\right\}^{-1},
\end{aligned}
$$

and (3.1) follows from (3.5). (3.2) and (3.3) are deduced similarly.

It remains to prove (3.4). From (3.6) we get

$$
D_{0} D_{1} D_{2}=g_{0} g_{1} g_{2}\left(g_{0}^{3}+g_{1}^{3}+g_{2}^{3}\right)-\left(g_{0}^{3} g_{1}^{3}+g_{1}^{3} g_{2}^{3}+g_{2}^{3} g_{0}^{3}\right) \text {. }
$$

Now, by lemma 3

$$
g_{0}^{3}+g_{1}^{3}+g_{2}^{3}-3 g_{0} g_{1} g_{2}=\frac{\varphi\left(x^{3}\right)^{4}}{\varphi\left(x^{9}\right)} .
$$

From lemma 2 we find

We have

$$
h_{1}=-3 x \varphi\left(x^{9}\right)^{3}, \quad h_{2}=0 .
$$

$$
\begin{aligned}
\varphi(x)^{3}= & \left(g_{0}+g_{1}+g_{2}\right)^{3} \\
= & g_{0}^{3}+g_{1}^{3}+g_{2}^{3}+6 g_{0} g_{1} g_{2}+ \\
& +3\left(g_{0}^{2} g_{1}+g_{1}^{2} g_{2}+g_{2}^{2} g_{0}\right)+ \\
& +3\left(g_{0} g_{1}^{2}+g_{1} g_{2}^{2}+g_{2} g_{0}^{2}\right) .
\end{aligned}
$$


Hence, remembering the definitions of $g_{s}$ and $h_{s}$, we conclude

$$
\begin{aligned}
& g_{0}^{2} g_{1}+g_{1}^{2} g_{2}+g_{2}^{2} g_{0}=-x \varphi\left(x^{9}\right)^{3}, \\
& g_{0} g_{1}^{2}+g_{1} g_{2}^{2}+g_{2} g_{0}^{2}=0 .
\end{aligned}
$$

Multiplication of (3.11) and (3.12) yields

(3.13) $0=g_{0} g_{1} g_{2}\left(g_{0}{ }^{3}+g_{1}{ }^{3}+g_{2}{ }^{3}\right)+3 g_{0}{ }^{2} g_{1}{ }^{2} g_{2}{ }^{2}+g_{0}{ }^{3} g_{1}{ }^{3}+g_{1}{ }^{3} g_{2}{ }^{3}+g_{2}{ }^{3} g_{0}{ }^{3}$.

We then get, by adding (3.9) and (3.13)

$$
\begin{aligned}
D_{0} D_{1} D_{2} & =2 g_{0} g_{1} g_{2}\left(g_{0}^{3}+g_{1}^{3}+g_{2}^{3}\right)+3 g_{0}^{2} g_{1}^{2} g_{2}^{2} \\
& =2 g_{0} g_{1} g_{2}\left(g_{0}^{3}+g_{1}^{3}+g_{2}{ }^{3}-3 g_{0} g_{1} g_{2}\right)+9\left(g_{0} g_{1} g_{2}\right)^{2} \\
& =2 x^{3} \frac{\varphi\left(x^{3}\right)^{5} \varphi\left(x^{27}\right)^{3}}{\varphi\left(x^{9}\right)^{2}}+9 x^{6} \frac{\varphi\left(x^{3}\right)^{2} \varphi\left(x^{27}\right)^{6}}{\varphi\left(x^{9}\right)^{2}} .
\end{aligned}
$$

Here we have made use of (3.8) and (3.10). Now (3.4) follows by (3.5).

4. The case $q=5$. We shall prove the identities

$$
\begin{aligned}
\sum_{n=0}^{\infty} p(5 n+4) x^{n} & =5 \frac{\varphi\left(x^{5}\right)^{5}}{\varphi(x)^{6}} \quad \text { (Ramanujan), } \\
\sum_{n=0}^{\infty} p(5 n+1) x^{n} \sum_{n=0}^{\infty} p(5 n+2) x^{n} & =2 \frac{\varphi\left(x^{5}\right)^{4}}{\varphi(x)^{6}}+25 x \frac{\varphi\left(x^{5}\right)^{10}}{\varphi(x)^{12}} \\
\sum_{n=0}^{\infty} p(5 n) x^{n} \sum_{n=0}^{\infty} p(5 n+3) x^{n} & =3 \frac{\varphi\left(x^{5}\right)^{4}}{\varphi(x)^{6}}+25 x \frac{\varphi\left(x^{5}\right)^{10}}{\varphi(x)^{12}}
\end{aligned}
$$

$$
\begin{aligned}
3 \sum_{n=0}^{\infty} p(5 n+1) x^{n} \sum_{n=0}^{\infty} p(5 n+2) x^{n}-2 \sum_{n=0}^{\infty} p(5 n) x^{n} \sum_{n=0}^{\infty} p(5 n+3) x^{n} \\
=x\left(\sum_{n=0}^{\infty} p(5 n+4) x^{n}\right)^{2},
\end{aligned}
$$

$$
\begin{gathered}
\sum_{n=0}^{\infty}\left\{p(5 n+1) x^{5 n}+p(5 n+2) x^{5 n+1}\right\} \\
=\frac{1}{\varphi\left(x^{5}\right)^{6}}\left\{\varphi(x)^{3} \varphi\left(x^{25}\right)^{2}+5 x \varphi(x)^{2} \varphi\left(x^{25}\right)^{3}+10 x^{2} \varphi(x) \varphi\left(x^{25}\right)^{4}+10 x^{3} \varphi\left(x^{25}\right)^{5}\right\}
\end{gathered}
$$

$$
\sum_{n=0}^{\infty}\left\{p(5 n) x^{5 n}+p(5 n+3) x^{5 n+3}\right\}
$$

$\frac{1}{\varphi\left(x^{5}\right)^{6}}\left\{\varphi(x)^{4} \varphi\left(x^{25}\right)+4 x \varphi(x)^{3} \varphi\left(x^{25}\right)^{2}+10 x^{2} \varphi(x)^{2} \varphi\left(x^{25}\right)^{3}+15 x^{3} \varphi(x) \varphi\left(x^{25}\right)^{4}+10 x^{4} \varphi\left(x^{25}\right)^{5}\right\}$. 
We shall also deduce four of the congruences of Atkin and SwinnertonDyer, viz.

$$
\sum_{n=0}^{\infty} p(5 n) x^{n} \equiv \varphi(x) \prod_{m=1}^{\infty}\left\{\left(1-x^{5 m-4}\right)\left(1-x^{5 m-1}\right)\right\}^{-3} \quad(\bmod 5),
$$$$
\sum_{n=0}^{\infty} p(5 n+1) x^{n} \equiv \varphi\left(x^{5}\right) \prod_{m=1}^{\infty}\left\{\left(1-x^{5 m-4}\right)\left(1-x^{5 m-1}\right)\right\}^{-1} \quad(\bmod 5) \text {, }
$$
$\sum_{n=0}^{\infty} p(5 n+2) x^{n} \equiv 2 \varphi\left(x^{5}\right) \prod_{m=1}^{\infty}\left\{\left(1-x^{5 m-3}\right)\left(1-x^{5 m-2}\right)\right\}^{-1} \quad(\bmod 5)$,

(4.10) $\sum_{n=0}^{\infty} p(5 n+3) x^{n} \equiv 3 \varphi(x) \prod_{m=1}^{\infty}\left\{\left(1-x^{5 m-3}\right)\left(1-x^{5 m-2}\right)\right\}^{-3} \quad(\bmod 5)$.

Proofs. By lemma 1

$$
g_{1}=-x \varphi\left(x^{25}\right), \quad g_{3}=g_{4}=0 .
$$

From

we find

$$
\varphi(x)^{3}=\left(g_{0}+g_{1}+g_{2}\right)^{3}
$$

$$
h_{2}=3 g_{0}^{2} g_{2}+3 g_{1}^{2} g_{0} \text {. }
$$

But $h_{2}=0$, by lemma 2. Hence

$$
g_{0} g_{2}+g_{1}^{2}=0 \text {. }
$$

It is convenient to use the following notation:

$$
\alpha=g_{1}^{-1} g_{0}, \quad \beta=g_{1}^{-1} g_{2}, \quad A=g_{1}^{-5} D, \quad A_{s}=g_{1}^{-4} D_{s} .
$$

From (4.12) we now get

$$
\alpha \beta=-1
$$

and by (4.11)

$$
\alpha+\beta=-\frac{\varphi(x)}{x \varphi\left(x^{25}\right)}-1 .
$$

Further, using (4.13) we find

Hence, by lemma 3

$$
A=\left|\begin{array}{lllll}
\alpha & 1 & \beta & 0 & 0 \\
0 & \alpha & 1 & \beta & 0 \\
0 & 0 & \alpha & 1 & \beta \\
\beta & 0 & 0 & \alpha & 1 \\
1 & \beta & 0 & 0 & \alpha
\end{array}\right|=\alpha^{5}+\beta^{5}+11
$$




$$
\alpha^{5}+\beta^{5}=-\frac{\varphi\left(x^{5}\right)^{6}}{x^{5} \varphi\left(x^{25}\right)^{6}}-11
$$

From lemma 4 we obtain

$$
\sum_{n=0}^{\infty} p(5 n+s) x^{5 n+s}=x^{4} \frac{\varphi\left(x^{25}\right)^{5}}{\varphi\left(x^{5}\right)^{6}} A_{s} .
$$

We then evaluate the determinants $A_{s}$ and find

$$
\begin{aligned}
& A_{0}=\alpha^{4}-3 \beta, \\
& A_{1}=-a^{3}+2 \beta^{2}, \\
& A_{2}=2 \alpha^{2}-\beta^{3}, \\
& A_{3}=-3 \alpha+\beta^{4}, \\
& A_{4}=5 .
\end{aligned}
$$

We can now easily prove the identities (4.1)-(4.6):

(4.1) follows immediately from (4.16) and (4.21).

Multiplication of (4.18) and (4.19) yields

Hence, by (4.15)

$$
A_{1} A_{2}=-2\left(\alpha^{5}+\beta^{5}\right)+3 \text {. }
$$

$$
A_{1} A_{2}=2 \frac{\varphi\left(x^{5}\right)^{6}}{x^{5} \varphi\left(x^{25}\right)^{6}}+25
$$

and (4.2) follows by (4.16). The proof of (4.3) is similar.

(4.4) follows directly from (4.1), (4.2) and (4.3).

Further

$$
\begin{aligned}
A_{1}+A_{2} & =-\left(\alpha^{3}+\beta^{3}\right)+2\left(\alpha^{2}+\beta^{2}\right) \\
& =-(\alpha+\beta)^{3}+2(\alpha+\beta)^{2}-3(\alpha+\beta)+4 .
\end{aligned}
$$

We insert for $\alpha+\beta$ from (4.14) and evaluate. Then (4.5) follows by (4.16).

Similarly

$$
\begin{aligned}
A_{0}+A_{3} & =\alpha^{4}+\beta^{4}-3(\alpha+\beta) \\
& =(\alpha+\beta)^{4}+4(\alpha+\beta)^{2}-3(\alpha+\beta)+2,
\end{aligned}
$$

and (4.6) follows in the same way.

We shall then prove the congruences (4.7)-(4.10). From (4.5) we get

$$
\sum_{n=0}^{\infty} p(5 n+1) x^{5 n} \equiv \frac{\varphi\left(x^{25}\right)^{2}}{\varphi\left(x^{5}\right)^{6}} h_{0} \quad(\bmod 5) .
$$

Now 


$$
\begin{aligned}
h_{0} & =\sum_{k=0}^{\infty}(-1)^{5 k}(10 k+1) x^{\frac{1}{2} \cdot 5 k(5 k+1)}+\sum_{k=1}^{\infty}(-1)^{5 k-1}(10 k-1) x^{\frac{1}{2}(5 k-1) 5 k} \\
& \equiv \sum_{k=-\infty}^{\infty}(-1)^{k} x^{\frac{1}{2} \cdot 5 k(5 k+1)}(\bmod 5) .
\end{aligned}
$$

We now make use of (3.7). Putting $y=-x^{5 / 2}, z=x^{25 / 2}$ we get

$$
h_{0} \equiv \varphi\left(x^{25}\right) \prod_{m=1}^{\infty}\left\{\left(1-x^{25 m-15}\right)\left(1-x^{25 m-10}\right)\right\} \quad(\bmod 5) \text {. }
$$

This can also be written

Hence, by (4.22)

$$
\left.h_{0} \equiv \varphi\left(x^{5}\right) \prod_{m=1}^{\infty}\left\{1-x^{25 m-20}\right)\left(1-x^{25 m-5}\right)\right\}^{-1} \quad(\bmod 5) .
$$

$$
\sum_{n=0}^{\infty} p(5 n+1) x^{n} \equiv \frac{\varphi\left(x^{5}\right)^{2}}{\varphi(x)^{5}} \prod_{m=1}^{\infty}\left\{\left(1-x^{5 m-4}\right)\left(1-x^{5 m-1}\right)\right\}^{-1}(\bmod 5) .
$$

But $\varphi(x)^{5} \equiv \varphi\left(x^{5}\right)(\bmod 5)$, and $(4.8)$ follows.

The remaining three congruences are now easily proved:

(4.9) follows directly from (4.2) and (4.8).

Further, by (4.17), (4.18) and (4.19)

Hence

$$
A_{0} A_{2}-2 A_{1}^{2}=15 \alpha-5 \beta^{4}
$$

and we get by (4.16)

$$
A_{0} \equiv 2 A_{1}^{2} A_{2}^{-1}(\bmod 5)
$$

$$
\sum_{n=0}^{\infty} p(5 n) x^{n} \equiv 2\left(\sum_{n=0}^{\infty} p(5 n+1) x^{n}\right)^{2}\left(\sum_{n=0}^{\infty} p(5 n+2) x^{n}\right)^{-1}(\bmod 5) \text {. }
$$

Now (4.7) follows by (4.8) and (4.9).

(4.10) follows from (4.3) and (4.7).

REMARK. Several relations involving the quantities $A_{s}$ can be established, which by (4.16) would yield other identities. For instance

$$
\begin{aligned}
& A_{0} A_{2}+A_{3} A_{4}=2 A_{1}^{2}, \\
& A_{1} A_{3}+A_{0} A_{4}=2 A_{2}^{2}, \\
& A_{0}^{2} A_{2}+A_{3}^{2} A_{1}=2\left(\alpha^{5}+\beta^{5}\right)^{2}+4\left(\alpha^{5}+\beta^{5}\right)+52, \\
& A_{1}^{2} A_{0}+A_{2}^{2} A_{3}=\left(\alpha^{5}+\beta^{5}\right)^{2}-13\left(\alpha^{5}+\beta^{5}\right)-14 .
\end{aligned}
$$

We also mention that elimination of $\alpha$ and $\beta$ from the equations (4.13), (4.14) and (4.15) would give us the Ramanujan-Watson modular equation of the 5th order (see Watson [14]). 
5. The case $q=7$. We have the identities

$$
\begin{aligned}
& \sum_{n=0}^{\infty} p(7 n+5) x^{n}=7 \frac{\varphi\left(x^{7}\right)^{3}}{\varphi(x)^{4}}+49 x \frac{\varphi\left(x^{7}\right)^{7}}{\varphi(x)^{8}} \quad \text { (Ramanujan) } \\
& \begin{aligned}
\sum_{n=0}^{\infty} p(7 n+1) x^{n} \sum_{n=0}^{\infty} p(7 n+3) x^{n} \sum_{n=0}^{\infty} p(7 n+4) x^{n} \\
=15 \frac{\varphi\left(x^{7}\right)^{5}}{\varphi(x)^{8}}+12 \cdot 7^{2} x \frac{\varphi\left(x^{7}\right)^{9}}{\varphi(x)^{12}}+24 \cdot 7^{3} x^{2} \frac{\varphi\left(x^{7}\right)^{13}}{\varphi(x)^{16}}+ \\
+3 \cdot 7^{5} x^{3} \frac{\varphi\left(x^{7}\right)^{17}}{\varphi(x)^{20}}+7^{6} x^{4} \frac{\varphi\left(x^{7}\right)^{21}}{\varphi(x)^{24}}
\end{aligned}
\end{aligned}
$$

$$
\begin{aligned}
\sum_{n=0}^{\infty} p(7 n) x^{n} \sum_{n=0}^{\infty} p(7 n+2) x^{n} \sum_{n=0}^{\infty} p(7 n+6) x^{n} & \\
=22 \frac{\varphi\left(x^{7}\right)^{5}}{\varphi(x)^{8}}+ & 2 \cdot 7^{3} x \frac{\varphi\left(x^{7}\right)^{9}}{\varphi(x)^{12}}+25 \cdot 7^{3} x^{2} \frac{\varphi\left(x^{7}\right)^{13}}{\varphi(x)^{16}}+ \\
& +3 \cdot 7^{5} x^{3} \frac{\varphi\left(x^{7}\right)^{17}}{\varphi(x)^{20}}+7^{6} x^{4} \frac{\varphi\left(x^{7}\right)^{21}}{\varphi(x)^{24}}
\end{aligned}
$$

Proofs. The lemmas 1 and 2 give

From

we find

$$
\begin{array}{ll}
g_{2}=-x^{2} \varphi\left(x^{49}\right), & g_{3}=g_{4}=g_{6}=0, \\
h_{6}=-7 x^{6} \varphi\left(x^{49}\right)^{3}, & h_{2}=h_{4}=h_{5}=0 .
\end{array}
$$

$$
\varphi(x)^{3}=\left(g_{0}+g_{1}+g_{2}+g_{5}\right)^{3}
$$

We put

$$
\alpha=g_{2}^{-1} g_{0}, \quad \beta=g_{2}^{-1} g_{1}, \quad \gamma=g_{2}^{-1} g_{5}, \quad A=g_{2}^{-7} D, \quad A_{s}=g_{2}^{-6} D_{s} .
$$

Now, from the equations (5.4)-(5.7) we obtain

$$
\alpha \beta^{2}+\alpha^{2}+\gamma=0, \quad \beta \gamma^{2}+\beta^{2}+\alpha=0, \quad \gamma \alpha^{2}+\gamma^{2}+\beta=0,
$$

$$
\alpha \beta \gamma=1 \text {. }
$$

By lemma 3

$$
A=-\frac{\varphi\left(x^{7}\right)^{8}}{x^{14} \varphi\left(x^{49}\right)^{8}}
$$


On the other hand, using (5.9), we find by evaluation of the determinant (5.11) $A=\alpha^{7}+\beta^{7}+\gamma^{7}-7\left(\alpha \beta^{5}+\beta \gamma^{5}+\gamma \alpha^{5}\right)+14\left(\alpha^{2} \beta^{3}+\beta^{2} \gamma^{3}+\gamma^{2} \alpha^{3}\right)+8$. It is now convenient to introduce quantities $y_{s}$ defined by

$$
y_{1}=\alpha^{3} \beta, \quad y_{2}=\beta^{3} \gamma, \quad y_{3}=\gamma^{3} \alpha .
$$

Then, by (5.8) and (5.9) we easily find

$$
y_{1} y_{2}=-y_{1}-1, \quad y_{2} y_{3}=-y_{2}-1, \quad y_{3} y_{1}=-y_{3}-1
$$

(5.15) $\alpha^{2} \beta^{3}=-y_{1}-1$,

$$
y_{1} y_{2} y_{3}=1
$$

(5.16) $\alpha \beta^{5}=y_{1}-y_{2}+1$,

$$
\beta^{2} \gamma^{3}=-y_{2}-1, \quad \gamma^{2} \alpha^{3}=-y_{3}-1,
$$

$$
\alpha^{7}=-y_{1}^{2}+y_{1}-y_{3}-1, \beta^{7}=-y_{2}^{2}+y_{2}-y_{1}-1 \text {, }
$$

Now, returning to (5.11) we get

$$
\gamma^{7}=-y_{3}^{2}+y_{3}-y_{2}-1 \text {. }
$$

$$
\begin{aligned}
A & =-\left(y_{1}^{2}+y_{2}^{2}+y_{3}^{2}\right)-14\left(y_{1}+y_{2}+y_{3}\right)-58 \\
& =-\left(y_{1}+y_{2}+y_{3}\right)^{2}-16\left(y_{1}+y_{2}+y_{3}\right)-64 \\
& =-\left(y_{1}+y_{2}+y_{3}+8\right)^{2} .
\end{aligned}
$$

Hence, by (5.10)

$$
y_{1}+y_{2}+y_{3}+8= \pm \frac{\varphi\left(x^{7}\right)^{4}}{x^{7} \varphi\left(x^{49}\right)^{4}}
$$

Considering the first term in the expansions of $\alpha, \beta$ and $\gamma$ in powers of $x$, we find

$$
y_{1}=-x^{-7}+\ldots, \quad y_{2}=-1+\ldots, \quad y_{3}=x^{7}+\ldots .
$$

We therefore have to take the sign - in (5.18). With the abbreviation

$$
T=\frac{\varphi\left(x^{7}\right)^{4}}{x^{7} \varphi\left(x^{49}\right)^{4}}
$$

we thus get

$$
y_{1}+y_{2}+y_{3}=-T-8
$$

Then, by (5.13)

$$
y_{1} y_{2}+y_{2} y_{3}+y_{3} y_{1}=T+5 \text {. }
$$

Together with (5.14) this enables us to express in terms of $T$ any symmetric polynomial in $y_{1}, y_{2}$ and $y_{3}$.

From lemma 4 we get 


$$
\sum_{n=0}^{\infty} p(7 n+s) x^{7 n+s}=x^{12} \frac{\varphi\left(x^{49}\right)^{7}}{\varphi\left(x^{7}\right)^{8}} A_{s} .
$$

Evaluation of the determinants $A_{s}$ gives

(5.24) $A_{1}=-\alpha^{5} \beta+\beta^{2} \gamma^{4}-\gamma^{3} \alpha^{3}+2 \gamma^{4} \alpha-3 \beta^{3} \gamma^{2}+\beta^{4}-6 \alpha \beta^{2}+2 \alpha^{2}-3 \gamma$ ，

(5.25) $A_{2}=\gamma^{6}+\alpha^{4} \beta^{2}-\alpha^{5}-5 \beta \gamma^{4}+4 \gamma \alpha^{3}+6 \beta^{2} \gamma^{2}+\beta^{3}+\gamma^{2} \alpha$,

(5.26) $A_{3}=-\beta^{5} \gamma+\gamma^{2} \alpha^{4}-\alpha^{3} \beta^{3}+2 \alpha^{4} \beta-3 \gamma^{3} \alpha^{2}+\gamma^{4}-6 \beta \gamma^{2}+2 \beta^{2}-3 \alpha$,

(5.27) $A_{4}=-\gamma^{5} \alpha+\alpha^{2} \beta^{4}-\beta^{3} \gamma^{3}+2 \beta^{4} \gamma-3 \alpha^{3} \beta^{2}+\alpha^{4}-6 \gamma \alpha^{2}+2 \gamma^{2}-3 \beta$,

(5.28) $\quad A_{5}=-\left(\alpha \beta^{5}+\beta \gamma^{5}+\gamma \alpha^{5}\right)+4\left(\alpha^{2} \beta^{3}+\beta^{2} \gamma^{3}+\gamma^{2} \alpha^{3}\right)-$

$$
-3\left(\alpha^{3} \beta+\beta^{3} \gamma+\gamma^{3} \alpha\right)+8,
$$

(5.29) $A_{6}=\beta^{6}+\gamma^{4} \alpha^{2}-\gamma^{5}-5 \alpha \beta^{4}+4 \beta \gamma^{3}+6 \alpha^{2} \beta^{2}+\alpha^{3}+\beta^{2} \gamma$.

We can now prove the Ramanujan identity (5.1): From (5.28) we get by (5.12), (5.15) and (5.16)

$$
A_{5}=-7\left(y_{1}+y_{2}+y_{3}\right)-7 \text {. }
$$

Hence, by (5.19) and (5.20)

$$
A_{5}=7 \frac{\varphi\left(x^{7}\right)^{4}}{x^{7} \varphi\left(x^{49}\right)^{4}}+49,
$$

and (5.1) follows from (5.22).

The proof of (5.2) is more complicated. From (5.24) we find

$$
\beta^{3} A_{1}=y_{1}^{2}-5 y_{2}^{2}-8 y_{1}+y_{2}-12,
$$

and then, by (5.13),

$$
y_{1}^{2} \beta^{3} A_{1}=y_{1}^{4}-8 y_{1}^{3}-18 y_{1}^{2}-11 y_{1}-5 .
$$

Denoting this polynomial by $Q$ we have

$$
y_{1}^{2} \beta^{3} A_{1}=Q\left(y_{1}\right) \text {. }
$$

In the same way we find

$$
\begin{aligned}
& y_{2}{ }^{2} \gamma^{3} A_{3}=Q\left(y_{2}\right), \\
& y_{3}{ }^{2} \alpha^{3} A_{4}=Q\left(y_{3}\right)
\end{aligned}
$$

Now, by multiplication we get

$$
A_{1} A_{3} A_{4}=Q\left(y_{1}\right) Q\left(y_{2}\right) Q\left(y_{3}\right)
$$

that is, a polynomial of degree 12 , symmetrical in $y_{1}, y_{2}$ and $y_{3}$. From 
(5.20), (5.21) and (5.14) we then conclude that $A_{1} A_{3} A_{4}$ is expressible as a polynomial in $T$, of degree 12 at most:

$$
A_{1} A_{3} A_{4}=\sum_{j=0}^{12} a_{j} T^{j}
$$

The coefficients $a_{j}$ could of course be computed directly, but the following method is simpler. From (5.22) and (5.30) we get

$$
\sum_{n=0}^{\infty} p(7 n+1) x^{n} \sum_{n=0}^{\infty} p(7 n+3) x^{n} \sum_{n=0}^{\infty} p(7 n+4) x^{n}=\sum_{j=0}^{12} a_{j} x^{4-j} \frac{\varphi\left(x^{7}\right)^{21-4 j}}{\varphi(x)^{24-4 j}}
$$

We thus immediately conclude that

$$
a_{12}=a_{11}=\ldots=a_{5}=0 .
$$

The remaining five $a_{j}$ 's can now be found by evaluating the first five terms in the power series expansions of the two sides of (5.31), and comparing the coefficients. Thus (5.2) is established.

The proof of (5.3) is quite analogous.

6. The case $q=2$. For the sake of completeness we add the following identities with $q=2$, although they are rather trivial:

$$
\begin{aligned}
& =\frac{\varphi\left(x^{2}\right) \varphi\left(x^{24}\right)}{\varphi(x)^{3}}\left(\prod_{m=1}^{\infty}\left\{\left(1+x^{24 m-13}\right)\left(1+x^{24 m-11}\right)\right\}-x \prod_{m=1}^{\infty}\left\{\left(1+x^{24 m-19}\right)\left(1+x^{24 m-5}\right)\right\}\right), \\
& \quad \sum_{n=0}^{\infty} p(2 n+1) x^{n} \\
& =\frac{\varphi\left(x^{2}\right) \varphi\left(x^{24}\right)}{\varphi(x)^{3}}\left(\prod_{m=1}^{\infty}\left\{\left(1+x^{24 m-17}\right)\left(1+x^{24 m-7}\right)\right\}-x^{2} \prod_{m=1}^{\infty}\left\{\left(1+x^{24 m-23}\right)\left(1+x^{24 m-1}\right)\right\}\right) .
\end{aligned}
$$

Proofs. By lemma 4

Now

$$
\sum_{n=0}^{\infty} p(2 n+s) x^{2 n+s}=(-1)^{s} \frac{\varphi\left(x^{4}\right)}{\varphi\left(x^{2}\right)^{3}} g_{s} .
$$

$$
g_{0}=\sum_{k=-\infty}^{\infty}\left\{x^{2 k(12 k+1)}-x^{(4 k+1)(6 k+2)}\right\}
$$

and using (3.7) we easily find

$$
g_{0}=\varphi\left(x^{48}\right)\left(\prod_{m=1}^{\infty}\left\{\left(1+x^{48 m-26}\right)\left(1+x^{48 m-22}\right)\right\}-x^{2} \prod_{m=1}^{\infty}\left\{\left(1+x^{48 m-38}\right)\left(1+x^{48 m-10}\right)\right\}\right) .
$$

Hence (6.1) follows. The proof of (6.2) is similar. 
7. Additional remarks. Atkin and Swinnerton-Dyer [1] have shown that the quantities $g_{s}$, for $q>3$, can always be expressed by certain infinite products. For instance, if $q=5$, we have (this special result was stated by Ramanujan [13], and first proved by Darling [4]):

$$
\begin{aligned}
& g_{0}=\varphi\left(x^{5}\right) \prod_{m=1}^{\infty}\left\{\left(1-x^{25 m-20}\right)\left(1-x^{25 m-5}\right)\right\}^{-2}, \\
& g_{2}=-x^{2} \varphi\left(x^{5}\right) \prod_{m=1}^{\infty}\left\{\left(1-x^{25 m-15}\right)\left(1-x^{25 m-10}\right)\right\}^{-2} .
\end{aligned}
$$

By (4.17)-(4.20) and (4.16) we thus get four identities with $q=5$, similar to the identities (3.1)-(3.3). One of them is:

$$
\begin{gathered}
\sum_{n=0}^{\infty} p(5 n) x^{n} \\
=\frac{\varphi\left(x^{5}\right)}{\varphi(x)^{2}} \prod_{m=1}^{\infty}\left\{\left(1-x^{5 m-4}\right)\left(1-x^{5 m-1}\right)\right\}^{-8}-3 x \frac{\varphi\left(x^{5}\right)^{6}}{\varphi(x)^{7}} \prod_{m=1}^{\infty}\left\{\left(1-x^{5 m-4}\right)\left(1-x^{5 m-1}\right)\right\}^{2} .
\end{gathered}
$$

From lemma 4 we now conclude that identities of this kind always exist, but when $q>5$ they become much more complicated.

\section{BIBLIOGRAPHY}

1. A. O. L. Atkin and P. Swinnerton-Dyer, Some properties of partitions, Proc. London Math. Soc. (3) 4 (1954), 84-106.

2. W. N. Bailey, A note on two of Ramanujan's formulae, Quart. J. Math., Oxford Ser. (2) 3 (1952), 29-31.

3. W. N. Bailey, A further note on two of Ramanujan's formulae, Quart. J. Math., Oxford Ser. (2) 3 (1952), 158-160.

4. H. B. C. Darling, Proofs of certain identities and congruences enunciated by S. Ramanujan, Proc. London Math. Soc. (2) 19 (1921), 350-372.

5. N. J. Fine, On a system of modular functions connected with the Ramanujan identities, Tôhoku Math. J. (2) 8 (1956), 149-164.

6. H. Gupta, A table of partitions, Proc. London Math. Soc. (2) 39 (1935), 142-149.

7. D. Kruyswijk, On some well-known properties of the partition function $p(n)$ and Euler's infinite product, Nieuw Arch. Wiskunde (2) 23 (1950), 97-107.

8. J. Lehner, Ramanujan identities involving the partition function for the moduli $11^{\alpha}$, Amer. J. Math. 65 (1943), 492-520.

9. L.J. Mordell, Note on certain modular relations considered by Messrs. Ramanujan, Darling and Rogers, Proc. London Math. Soc. (2) 20 (1922), 408-416.

10. M. Newman, Remarks on some modular identities, Trans. Amer. Math. Soc. 73 (1952), 313-320.

11. H. Rademacher, The Ramanujan identities under modular substitutions, Trans. Amer. Math. Soc. 51 (1942), 609-636. 
12. H. Rademacher and H. S. Zuckerman, $A$ new proof of two of Ramanujan's identities, Ann. of Math. (2) 40 (1939), 473-489.

13. S. Ramanujan, Some properties of $p(n)$, the number of partitions of $n$, Proc. Cambridge Philos. Soc. 19 (1919), 207-210 (= Collected papers, 210-213).

14. G. N. Watson, Ramanujans Vermutung über Zerfällungsanzahlen, J. Reine Angew. Math. 179 (1938), 97-128.

15. H. S. Zuckerman, Identities analogous to Ramanujan's identities involving the partition function, Duke Math. J. 5 (1939), 88-110.

UNIVERSITY OF OSLO, NORWAY 